\title{
DOSSIER
}

\section{Retinopatia diabética - o papel da Medicina Geral e Familiar}

\author{
VANDA NOGUEIRA, * PEDRO MOURO, ${ }^{*}$ MARTA VILA-FRANCA, ${ }^{*}$ \\ MARGARIDA MESQUITA, ${ }^{* *}$ PAULO CALDEIRA-ROSA, ${ }^{* * *}$ ROSA GALLEGO, ${ }^{* * * *}$ \\ ANTÓNIO CASTANHEIRA-DINIS*****
}

\section{RESUMO}

Introduccão: A retinopatia diabética é uma das principais causas de cegueira e de disfunção visual nos países desenvolvidos, sendo a prevenção do desenvolvimento e progressão das lesões oculares um dos principais objectivos a atingir pela equipa de Medicina Geral e Familiar/Oftalmologia. Métodos: No presente trabalho foi realizada uma revisão de variadas publicações recentes, de centros conceituados, sobre os factores de risco para a retinopatia diabética e novos programas de rastreio, diagnóstico e terapêutica. A extensa investigação realizada nesta área tem proporcionado uma grande evolução, quer nos cuidados prestados aos doentes, quer no aumento do leque de métodos diagnósticos e ferapêuticos disponíveis, com consequente melhoria do prognóstico visual. Conclusão: Só o correcto acompanhamento do doente diabético pela equipa de Medicina Geral e Familiar/Oftalmologia permite a aplicação da terapêutica adequada em tempo útil, trazendo beneficios inquestionáveis para a funç̃õo visual.

Palavras chave: Diabetes Mellitus; Retinopatia Diabética. duzem a uma progressão da doença, bem como referenciando o doente a um especialista para observação e eventual tratamento.

A presente revisão irá abordar sistematizadamente a fisiopatologia, factores de risco, manifestações clinicas e opções terapêuticas disponiveis, procurando também fornecer conceitos práticos no que diz respeito essencialmente à prevenção primária e secundária da retinopatia diabética.

\section{FACTORES DE RISCO}

A DM divide-se em dois grupos distintos, com base nas características clínicas, etiologia e bases fisiopatológicas: a DM tipo 1 e tipo 2. Entre 90 e 95\% dos diabéticos sofrem de DM tipo 2, pelo que, não obstante a incidência e gravidade das complicações serem maiores no doente com DM tipo 1, o maior número de doentes afectados por retinopatia diabética, com repercussão visual significativa, está, naturalmente, no grupo dos doentes com doença do tipo 2.

A duração da doença constitui um factor de risco major associado ao desenvolvimento da retinopatia diabética. Após cinco anos, 25\% dos diabéticos tipo 1 têm retinopatia diabética. Dez anos depois do diagnóstico quase 60\% apresentam retinopatia e o número sobe para $80 \%$ aos 15 anos, sendo que quase $25 \%$ dos doentes sofrem da 


\section{DOSSIER}

forma mais agressiva e ameaçadora para a visão, a retinopatia diabética proliferativa (RDP).

Do grupo de doentes com DM tipo 2 com doença diagnosticada há menos de cinco anos, 24\% apresentam retinopatia, mas a percentagem sobe para $40 \%$ nos que são insulino-tratados. Estas taxas aumentam para 53\% e $84 \%$ respectivamente, após cerca de 20 anos. A RDP desenvolve-se em $2 \%$ dos doentes com doença documentada até há cinco anos, sendo a sua prevalência de aproximadamente 25\% após 25 anos. ${ }^{1}$

O nivel de glicémia constitui o principal factor de risco modificável relacionado com o desenvolvimento de retinopatia diabética. O estudo Diabetes Control and Complications Trial (DCCT) investigou o efeito do controlo intensivo da glicémia em doentes com diabetes tipo 1. Durante um follow-up médio de seis anos a terapia intensiva reduziu o risco de desenvolvimento de retinopatia em $76 \% .^{2} \mathrm{O}$ efeito protector do controlo glicémico foi também confirmado em doentes com DM tipo 2 no estudo United Kingdom Prospective Diabetes Study (UKPDS), o qual, à semelhança do DCCT, mostrou também benefício em relação ao risco de desenvolvimento de retinopatia diabética, bem como de nefropatia e de neuropatia. A taxa global de complicações microvasculares foi reduzida em cerca de $25 \%$. O UKPDS procurou também estabelecer a influência do controlo da Pressão Arterial (PA) na retinopatia diabética e, após um follow-up de cerca de 8,4 anos, verificou-se uma redução de $34 \%$ na progressão da retinopatia, com uma redução em cerca de $47 \%$ do risco de deterioração da acuidade visual. ${ }^{3}$

A gravidez é, também, um factor de risco major para a progressão da retinopatia diabética, sobretudo no fim do segundo trimestre e princípio do terceiro. Os estudos publicados apontam para um risco de progressão da ordem dos $25 \% .{ }^{4}$ Os factores que condicionam o comportamento da retinopatia diabética durante a gestação estão identificados, sendo os mais significativos a duração da diabetes, a gravidade da retinopatia no início da gravidez e o controle da glicémia e da PA durante a gestação. ${ }^{4}$ No entanto, a DM gestacional não aumenta o risco de desenvolvimento de retinopatia diabética. ${ }^{5}$

A dislipidemia tem sido associada a uma acumulação de exsudados duros na retina, o que, por sua vez, se tem relacionado com o aumento do risco de diminuição da acuidade visual. ${ }^{6}$ Alguns estudos referem apenas a associação deste factor de risco em diabéticos tipo $1 .{ }^{7}$ Novos estudos científicos randomizados, a decorrer, trarão mais informação sobre o papel da dislipidemia na incidência e progressão da retinopatia diabética.

A obesidade central tem, também, sido apontada como factor de risco para o desenvolvimento de retinopatia. ${ }^{8} \mathrm{Ou}-$ tros factores estudados, como a idade, função renal, utilização de Inibidores da Enzima de Conversão da Angiotensina ou Antagonistas dos Receptores da Angiotensina 2, têm sido objecto de alguma controvérsia e ainda não foi provada qualquer influência no comportamento da retinopatia diabética. ${ }^{9}$

\section{HISTÓRIA NATURAL}

O estádio inicial da retinopatia diabética é a denominada retinopatia diabética não-proliferativa (RDNP), que se caracteriza por alterações vasculares retinianas, incluindo micro-aneurismas, hemorragias intra-retinianas e manchas algodonosas. As alterações ao nível da permeabilidade vascular, devido à ruptura da barreira hemato-retiniana, podem resultar em qualquer fase do espectro da retinopatia diabética, com aumento da espessura da retina (edema) e depósitos lipídicos (exsudatos duros). No caso deste edema retiniano obe- 
decer a determinados critérios de gravidade e progressão (Edema Macular Clinicamente Significativo), poderá estar indicada a fotocoagulação laser focal. ${ }^{10}$

À medida que a doença progride, ocorre uma oclusão gradual a nivel microvascular, a qual resulta em fenómenos de má perfusão e isquémia da retina. Os sinais de isquémia progressiva incluem alterações venosas (veias em rosário, loops vasculares), anomalias microvasculares intra-retinianas (IRMA), bem como agravamento da exsudação com aumento progressivo do edema retiniano e dos depósitos lipídicos. Neste estádio, conhecido, de acordo com critérios definidos, como RDNP grave, ou pré-proliferativa, poderá ser considerada a terapêutica laser na forma de fotocoagulação panretiniana.

Os estádios mais avançados da doença incluem-se na categoria da RDP e caracterizam-se pelo início do desenvolvimento de neovascularização a nível do disco óptico e da superficie interna da retina (Figura 1A). Estes neovasos encontram-se sujeitos a hemorragia para o interior da cavidade vitrea, hemovítreo, com consequente diminuição súbita da acuidade visual de grau variável. Poderá ocorrer também fibrose e contracção destas estruturas, com a formação de membranas pré-retinianas, rasgaduras e descolamento traccional ou regmatógeno da retina. Os neovasos poderão ainda surgir a nível do estroma da îris, estendendo-se ao ângulo da câmara anterior, por onde ocorre a drenagem de humor aquoso, com aumento da pressão intra-ocular, dando origem a um glaucoma secundário de prognóstico sombrio.

\section{Rastreio e Avallaç̃ó DA RETINOPATIA DiabítICA}

As complicações visuais da DM podem e devem ser evitadas ou controladas. Dois estudos científicos publicados mostram que o tratamento da retinopatia diabética pode ser eficaz na prevenção da perda visual severa (acuidade visual $<5 / 200$ ) em $90 \%$ dos casos. ${ }^{11}$ No entanto, apesar de existirem actualmente indicações concretas para a observação oftalmológica dos doentes diabéticos e de estarem disponíveis estratégias terapêuticas eficazes, nem todos os doentes têm acesso em tempo útil às potencialidades da Oftalmologia.

O papel da Medicina Geral e Familiar é, então, fundamental, quer para o controle metabólico e dos factores de risco associados, quer para a referenciação a outras especialidades, em particular a Oftalmologia, para observação e planeamento do seguimento destes doentes.

Aconselha-se que os doentes com DM tipo 1 sejam referenciados ao oftalmologista de imediato se existir patologia oftalmológica conhecida ou, caso contrário, cinco anos após o diagnóstico de DM. A retinopatia com risco para a função visual é rara em doentes com DM tipo 1 antes da puberdade e nos primeiros 3-5 anos da doença. ${ }^{12}$ Propõe-se, por isso, que as observações oftalmológicas tenham início cinco anos após o diagnóstico de DM tipo 1 e anualmente se não existirem alterações. Por outro lado, a data do início da DM tipo 2 é de dificil determinação e frequentemente precede o diagnóstico em alguns anos. Sabe-se que aproximadamente 30\% destes doentes têm manifestações de retinopatia diabética na altura do diagnóstico e 3\% apresentam já critérios de gravidade. ${ }^{13} \mathrm{~A}$ observação oftalmológica deverá, por isso, ser realizada logo que se diagnostique DM e anualmente se não surgirem alterações.

As doentes diabéticas que planeiem engravidar deverão realizar uma observação oftalmológica pré-concepcional, em que será dada informação detalhada sobre o risco de desenvolvimento e/ou progressão da retinopatia e planeado o seguimento oftalmológico adequado. 


\section{DOSSIER}

Todas as grávidas deverão realizar um exame oftalmológico durante o primeiro trimestre, sendo subsequentemente observadas segundo um plano proposto pelo oftalmologista e que se baseará na presença e gravidade das lesões retinianas e no controle metabólico conseguido. O seguimento de uma grávida com DM só termina com uma observação pós-parto, em que não se verifiquem alterações. As grávidas com o diagnóstico de DM gestacional não necessitam de observação oftalmológica de rotina durante a gravidez. ${ }^{5} \mathrm{O}$ Quadro I resume o plano proposto para as avaliações oftalmológicas do doente diabético. ${ }^{11}$

Dada a dificuldade de acesso de todos os doentes diabéticos a consultas periódicas de Oftalmologia, têm sido iniciados programas de rastreio de retinopatia diabética, baseados na realização de retinografias (fotografias digitais da retina), de acordo com o plano apresentado no Quadro 1. As retinografias são posteriormente analisadas por um Médico Oftalmologista e sempre que se verificam alterações é marcada consulta de Oftalmologia consoante a gravidade das mesmas. A calendarização das retinografias é realizada de acordo com o plano apresentado. Este método de rastreio não deverá substituir as consultas de Oftalmologia durante a gravidez.

A pesquisa e avaliação das lesões de retinopatia diabética é realizada em consulta de Oftalmologia onde é efectuada uma anamnese cuidada, permitindo a estimativa do risco de existên- cia ou progressão das lesões. A observação oftalmológica inclui avaliação da acuidade visual, da pressão intra-ocular e observação do segmento anterior e do segmento posterior do globo ocular após midríase farmacológica. Se necessário são realizados Exames Complementares de Diagnóstico (ECD) como a angiografia fluoresceínica, que permite o estudo da vascularização retiniana e coroideia após injecção de contraste iodado endovenoso (Figuras $1 \mathrm{~B} \mathrm{e}$ 1C). Um ECD recente é a Tomografia de Coerência Óptica (OCT) que avalia, com alta definição e utilizando uma técnica de interferometria, a região macular (Edema Macular) (Figuras 2 e 3).

O plano de follow-up de um doente será modificado sempre que surjam alterações que o justifique.

\section{TERAPÊUTICA}

O tratamento médico da retinopatia diabética depende essencialmente do controle metabólico. Estudos clínicos controlados e randomizados demonstraram que um controle rigoroso da glicemia para níveis próximos dos considerados normais atrasa o desenvolvimento e progressão da retinopatia, tanto na DM tipo 1 como na DM tipo $2 .^{14,15}$ Um agravamento transitório da retinopatia pode ser observado em doentes diabéticos do tipo 1 no período que segue à normalização glicémica. ${ }^{16}$ No entanto, raramente há progressão para

QUADRO I

\begin{tabular}{|c|c|c|}
\hline \multicolumn{3}{|c|}{ QUADRO I } \\
\hline \multicolumn{3}{|c|}{ PLANO PROPOSTO PARA AS AVALIAC̣̃̃ES OFTALMOLÓGICAS DO DOENTE DIABÉTICO } \\
\hline Tipo de Diabetes & Tempo Recomendado para o $1^{\circ}$ exame & Seguimento Aconselhado \\
\hline Tipo 1 & 5 anos após o início da doença & Anualmente \\
\hline Tipo 2 & Na altura do diagnóstico & Anualmente \\
\hline $\begin{array}{l}\text { Anterior a gravidez } \\
\text { (tipo l ou tipo 2) }\end{array}$ & 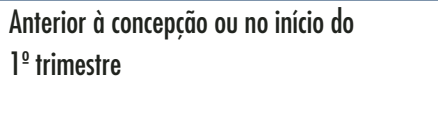 & $\begin{array}{l}\text { Sem retinopatia, ou retinopatia ligeira ou } \\
\text { moderada, todos os 3-12 meses; RDNP grave } \\
\text { ou com agravamento, todos os } 1-3 \text { meses }\end{array}$ \\
\hline
\end{tabular}




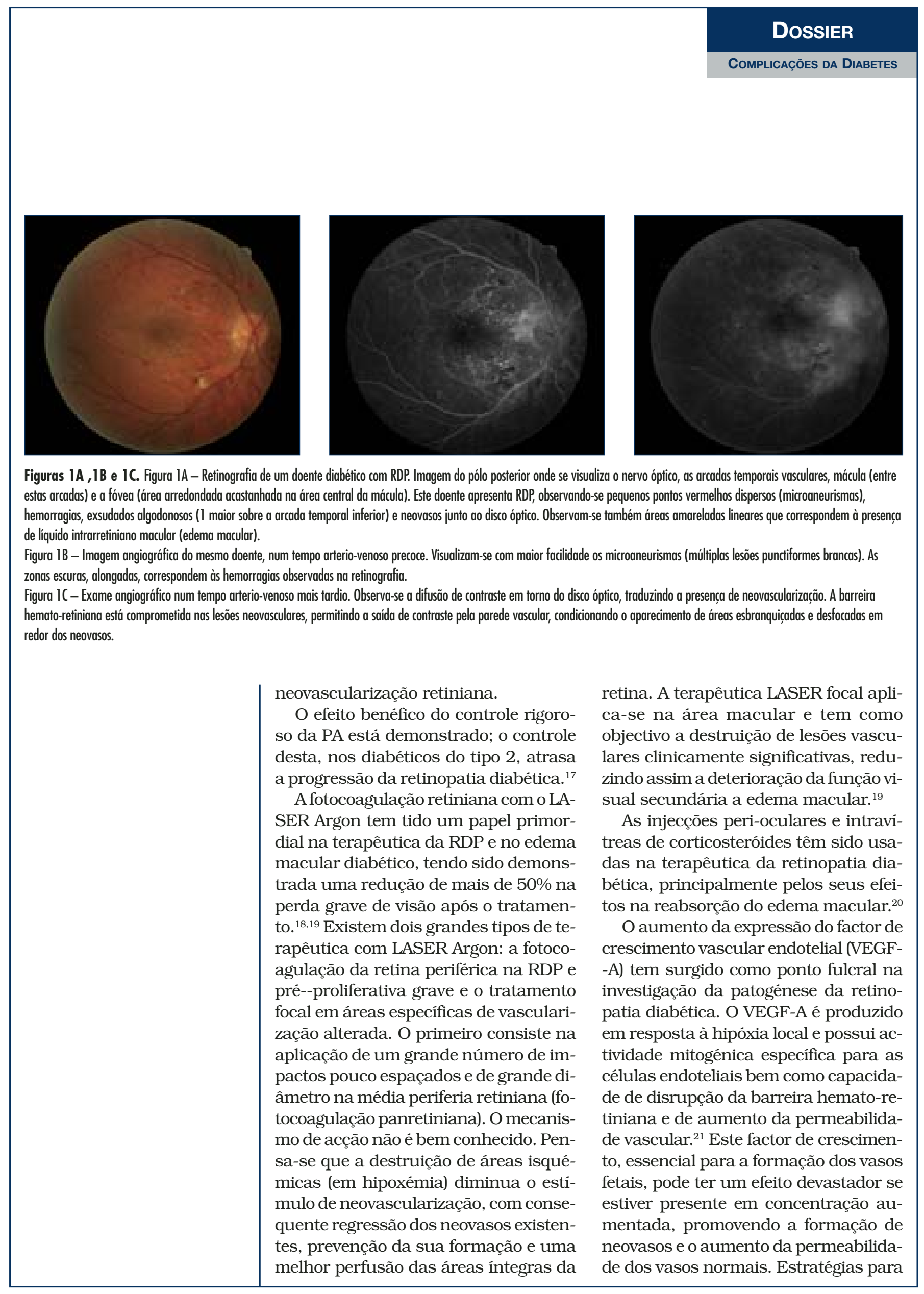




\section{DOSSIER}

Complicações da Diabetes

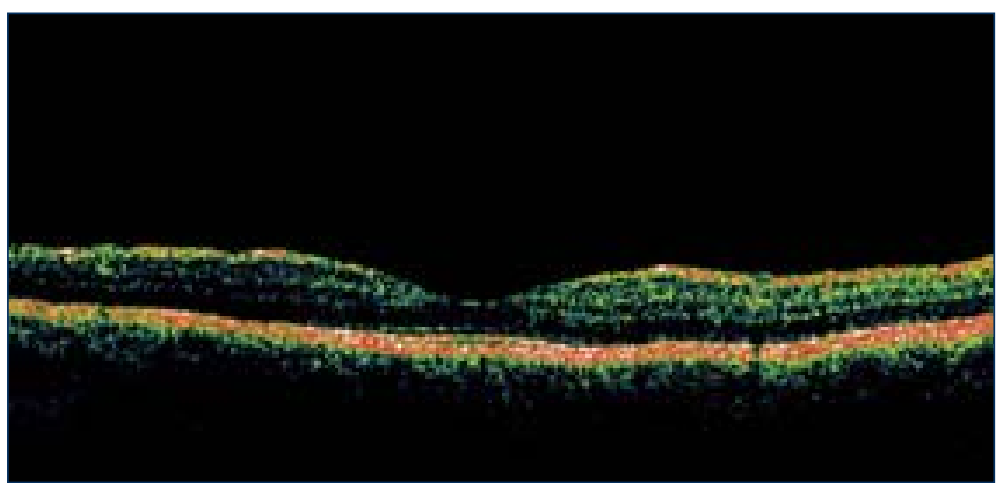

Figura 2. Imagem em secção da área macular da retina obtida por 0cT. Área macular sem alterações, com depressão normal na zona central da fóvea e sem espessamentos da retina.

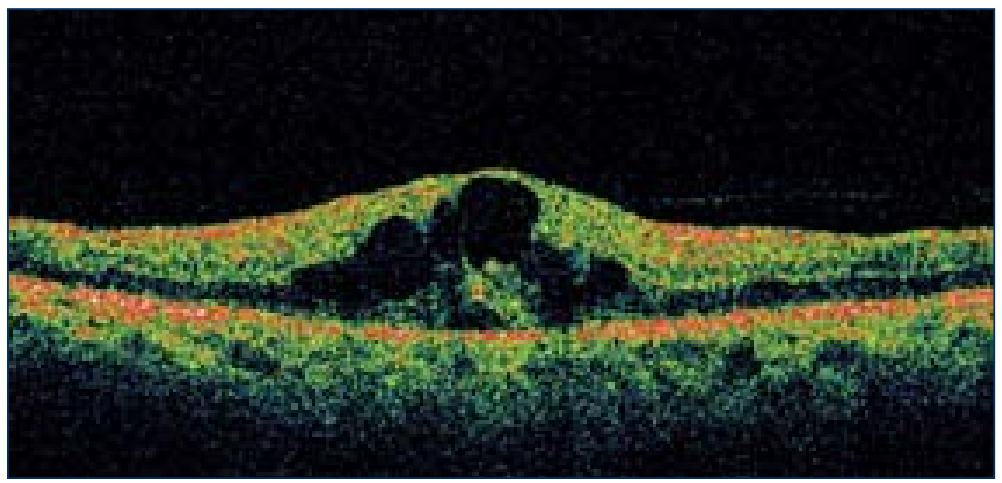

Figura 3. Imagem também obtida por OCT da área macular de um doente diabético com edema macular. Note-se o espessamento da área foveal, com perda da depressão foveolar e com áreas de menor reflectância (mais escuras) que correspondem a líquido intrarretiniano.

bloquear a formação de VEGF-A ou para prevenir a sua acção no globo ocular têm-se apresentado como terapêuticas promissoras na retinopatia diabética, sobretudo no edema macular diabético. No entanto, os efeitos sistémicos da terapêutica anti-VEGF podem ser graves, por exemplo ao nível dos vasos sanguíneos do miocárdio e dos membros inferiores, muitas vezes afectados nos doentes diabéticos. Tem-se optado, por isso, pela injecção intravitrea destes fármacos, diminuindo a sua concentração em circulação. Ainda não foram registados efeitos sistémicos graves com a terapêutica intravitrea mas faltam estudos para definir o perfil de segurança. Actualmente existem dois fármacos com acção
anti-VEGF, preparados para injecção intravitrea, o pegaptanib e o ranibizumab.

Doentes com retinopatia diabética mais avançada, como hemovitreo persistente e descolamento de retina traccional causada por bandas fibrosas que acompanham os neovasos, beneficiam de intervenção cirúrgica, a vitrectomia (Figuras 4A , 4B e 4C). ${ }^{22,23}$

O Quadro II sumariza o esquema de tratamento da retinopatia diabética recomendado pelo Programa Nacional para a Saúde da Visão. ${ }^{24}$

\section{CONCLUSÃO}

A retinopatia diabética é uma das prin- 


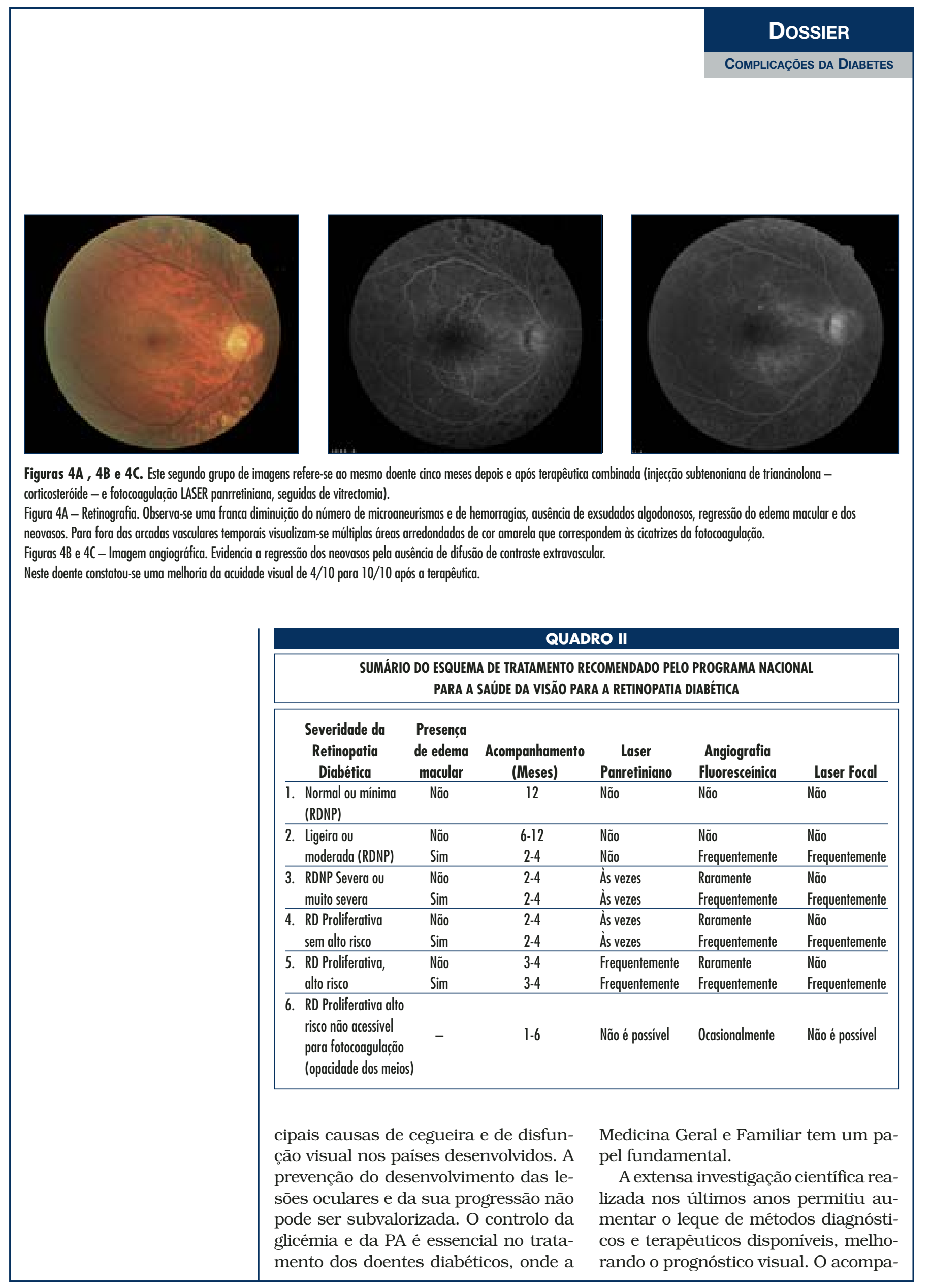


nhamento do doente diabético pela equipa de Medicina Geral e Familiar/ /Oftalmologia possibilita a aplicação da terapêutica adequada em tempo útil, trazendo benefícios inquestionáveis para a função visual.

\section{REFERÊNCIAS BIBLIOGRÁFICAS}

1. Chew EY, Benson WE, Boldt HC, Chang TS, Lobes Jr, LA, Miller JW, et al. American Academy of Ophthalmology Retina Panel: Preferred Practice Patterns. San Francisco, Ca: American Academy of Ophthalmology; 2003.

2. Fong DS, Aiello L, Gardner TW, King GL, Blankenship G, Cavallerano JD, et al; American Diabetes Association. Retinopathy in diabetes. Diabetes Care 2004 Jan; 27 Suppl 1: S84-7.

3. Fong DS, Ferris FL $3^{\text {rd }}$. Focal Points: clinical modules for ophthalmologists: practical management of diabetic retinopathy. San Francisco, CA: American Academy of Ophthalmology; 2003.

4. Chew EY, Mills JL, Metzger BE, Remaley NA, Jovanovic-Peterson L, Knopp RH, et al. Metabolic control and progression of retinopathy. The Diabetes in Early Pregnancy study (DIEP). Diabetes Care 1995 May; 18 (5):631-7.

5. Klein BE, Moss SE, Klein R. Effect of pregnancy on progression of diabetic retinopathy. Diabetes Care 1990 Jan; 13 (1): 34-40.

6. Chew EY, Klein ML, Ferris FL $3^{\text {rd }}$, Remaley NA, Murphy RP, Chantry K, et al. Association of elevated serum lipid levels with hard exudate in diabetic retinopathy. Early Treatment Diabetic Retinopathy Study (ETDRS) Report 22. Arch Ophthalmol 1996 Sep; 114 (9): 1079-84.

7. Klein BE, Moss SE, Klein R, Surawicz TS. The Wisconsin Epidemiologic Study of Diabetic Retinopathy. XIII: relationship of serum cholesterol to retinopathy and hard exudate. Ophthalmology 1991 Aug; 98 (8): 1261-5.

8. Wong TY, Klein R, Islam FM, Cotch MF, Folsom AR, Klein BE, et al. Diabetic retinopathy in a multi-ethnic cohort in the United States. Am J Ophthalmol 2006 Mar; 141 (3): 446-55.

9. Davis MD, Fisher MR, Gangnon RE, Barton F, Aiello LM, Chew EY, et al. Risk factors for high-risk proliferative diabetic retinopathy and severe visual loss: Early Treatment Diabetic Retinopathy Study Report \#18. Invest Ophthalmol Vis Sci 1998 Feb; 39 (2): 233-52.
10. Early Treatment Diabetic Retinopathy Study research group. Photocoagulation for diabetic macular edema. Early Treatment Diabetic Retinopathy Study report number 1 . Arch Ophthalmol 1985 Dec; 103 (12): 1796-806.

11. Ferris FL $3^{\text {rd }}$. How effective are treatments for diabetic retinopathy? JAMA 1993 Mar 10; 269 (10): 1290-1.

12. Krolewski AS, Warram JH, Rand LI, Christlieb AR, Busick EJ, Kahn CR. Risk of proliferative diabetic retinopathy in juvenile-onset type 1 diabetes: a 40-yr follow-up study. Diabetes Care 1986 Sep-Oct; 9 (5): 443-52.

13. Klein R, Klein BE, Moss SE, Davis MD, DeMets DL. The Wisconsin epidemiologic study of diabetic retinopathy. III. Prevalence and risk of diabetic retinopathy when age at diagnosis is 30 or more years. Arch Ophthalmol 1984 Apr; 102 (4): 527-32.

14. The Diabetes Control and Complications Trial Research Group. The effect of intensive treatment of diabetes on the development and progression of long-term complications in insulin-dependent diabetes mellitus. N Engl J Med 1993 Sep 30; 329 (14): 977-86.

15. UK Prospective Diabetes Study (UKPDS) Group. Intensive blood-glucose control with sulphonylureas or insulin compared with conventional treatment and risk of complications in patients with type 2 diabetes (UKPDS 33). Lancet 1998 Sep 12; 352 (9131): 837-53. Erratum in: Lancet 1999 Aug 14; 354(9178): 602.

16. Early worsening of diabetic retinopathy in the Diabetes Control and Complications Trial. Arch Ophthalmol 1998 Jul; 116 (7): 874-86. Erratum in: Arch Ophthalmol 1998 nov; 116 (1): 1469.

17. UK Prospective Diabetes Study (UKPDS) Group. Tight blood pressure control and risk of macrovascular and microvascular complications in type 2 diabetes: UKPDS 38. BMJ 1998 Sep 12; 317 (7160): 703-13. Erratum in: BMJ 1999 Jan 2; 318 (7175): 29.

18. Diabetic Retinopathy Study Research Group. Photocoagulation treatment of proliferative diabetic retinopathy: clinical application of Diabetic Retinopathy Study (DRS) findings, DRS report number 8. Ophthalmology 1981 Jul; 88 (7): 583-600.

19. Early Treatment Diabetic Retinopathy Study Research Group. Early photocoagulation for diabetic retinopathy: ETDRS report number 9. Ophthalmology 1991 May; 98 (5 suppl): 766-85.

20. Kocabora MS, Kucuksahin H, Gulkilik $\mathrm{G}$, et al. Traitement de l'oedème maculaire diabétique par injection intravitréenne d'aceto- 


\section{DOSSIER}

nide de triamcinolone: consequences fonctionnelles et anatomiques. J Fr Ophthalmol 2007; 30 (1): 32-8.

21. Ferrara N. Vascular endothelial growth factor: basic science and clinical progress. Endocr Rev 2004 Aug; 25 (4): 581-611.

22. The Diabetic Retinopathy Vitrectomy Study Research Group. Early vitrectomy for severe vitreous hemorrhage in diabetic retinopathy: two-year results of a randomized trial. Diabetic Retinopathy Vitrectomy Study report 2. Arch Ophthalmology 1985 Nov; 103 (11):
1644-52.

23. The Diabetic Retinopathy Vitrectomy Study Research Group. Early vitrectomy for severe proliferative diabetic retinopathy in eyes with useful vision: clinical application of results of a randomized trial. Diabetic Retinopathy Vitrectomy Study report 4. Ophthalmology 1988 Oct; 95 (10): 1321-34.

24. Principais Recomendações para o Diag nóstico e Tratamento da Retinopatia Diabética. Programa Nacional para a Saúde da Visão. Lisboa: Direcção-Geral da Saúde; 2007.

\section{DIABETIC RETINOPATHY - THE ROLE OF GENERAL AND FAMILY PRACTITIONER}

\section{ABSTRACT}

Introduction: Diabetic retinopathy is one of the main causes of blindness and of visual dysfunction in developed countries. The prevention of ocular lesions and their progression is the main objective of cooperation between General and Family Practitioners and Ophthalmologists.

Methods: In the present study we reviewed the most recent publications from major centres concerning risk factors of diabetic retinopathy, new screening programs, diagnosis and treatment. Research conducted in this area has produced not only great progress in the care of diabetic patients but also improvements in diagnostic methods and treatments and improvements in prognosis. Conclusion: Careful follow-up of diabetic patients by the General and Family Practitioner-Ophthalmologist team permits the use of adequate therapy at the right time, resulting in undeniable benefits for preservation of functional vision in diabetic patients.

Key words: Diabetes mellitus; Diabetic Retinopathy. 